\title{
A EFETIVIDADE DE AÇÕES EDUCATIVAS NA REDUÇÃO DE INCONFORMIDADES GERADAS PELO SISTEMA DRG-BRASIL EM IO HOSPITAIS PÚBLICOS DE MINAS GERAIS: ESTUDO DE CASO
}

\author{
Priscilla Rodrigues Fernandes de Oliveira \\ FHEMIG \\ priscillarfernandes@gmail.com \\ Cynthia Carolina Duarte Andrade \\ cynthiacarolinaduarteandrade@gmail.com
}

Victor Rodolfo Caldeira de Jesus victorrcjesus@gmail.com

Alisson Rubson Alves alisson.rubson@fhemig.mg.gov.br 


\section{RESUMO}

Objetivo: Descrever o impacto de ações educativas na redução de inconformidades apontadas pelo sistema Diagnosis Related Groups Brasil em I0 hospitais da Fhemig no período de janeiro a julho 202I. Referencial: O DRG- Brasil mensura e categoriza a complexidade e a gravidade clínica de pacientes e possibilita o levantamento de estratégias para melhor a assitência e alocação de recursos. Método: Estudo de caso sobre a efetividade de ações educativas na melhoria da qualidade da codificação realizada em IO hospitais da Fhemig através do software DRGBrasil. Foram realizadas reuniões mensais e treinamento com os analistas dos hospitais. A efetividade foi mensurada a partir da comparação do número de inconformidades geradas antes e após as ações educativas. Resultados: Das 23.326 altas codificadas no período da análise, foram geradas I.I22 inconformidades que após a ação educativa e reuniões realizadas reduziram em $64,4 \%$. Dentre os tipos de inconformidades, "CID com especialização não utilizada" foi a que apresentou maior ocorrência $(44,6 \%)$ e também maior redução $(69,0 \%)$. Conclusão: As ações educativas realizadas foram efetivas para melhoria da qualidade do processo de codificação.

Descritores: Grupos Diagnósticos Relacionados. Codificação Clínica. Sistema Único de Saúde.

\section{ABSTRACT}

Objective: To describe the impact of educational actions on the reduction of non-conformities pointed out by the Diagnosis Related Groups Brazil system in I0 Fhemig hospitals from January to July 202I. Reference: DRG-Brasil measures and categorizes the complexity and clinical severity of patients and enables the survey of strategies to improve assistance and resource allocation. Method: Case study on the effectiveness of educational actions in improving the quality of coding carried out in I0 hospitals of Fhemig through the DRG-Brasil software. Monthly meetings and training were held with hospital analysts. Effectiveness was measured by comparing the number of nonconformities generated before and after the educational actions. Results: Of the 23,326 discharges coded during the period of analysis, I,I22 nonconformities were generated, which after the educational action and meetings held, reduced by $64.4 \%$. Among the types of nonconformities, "ICD with unused specialization" was the one with the highest occurrence (44.6\%) and also the greatest reduction (69.0\%). Conclusion: The educational actions carried out were effective in improving the quality of the coding process.

Descriptors: Diagnosis-Related Groups. Clinical Coding. Unified Health System.

\section{RESUMEN}

Objetivo: Describir el impacto de las acciones educativas en la reducción de las no conformidades señaladas por el sistema Diagnóstico Grupos Relacionados Brasil en I0 hospitales de Fhemig de enero a julio de 202I. Referencia: DRG-Brasil mide y categoriza la complejidad y severidad clínica de los pacientes y permite el estudio de estrategias para mejorar la asistencia y la asignación de recursos. Método: Estudio de caso sobre la efectividad de las acciones educativas en la mejora de la calidad de la codificación realizada en IO hospitales de Fhemig a través del software DRG-Brasil. Se realizaron reuniones y capacitaciones mensuales con analistas hospitalarios. La efectividad se midió comparando el número de no conformidades generadas antes y después de las acciones educativas. Resultados: De las 23.326 altas codificadas en el período de análisis, se generaron I.I22 no conformidades, que luego de la acción educativa y reuniones realizadas, se redujeron en un $64,4 \%$. Entre los tipos de no conformidades, el "DAI con especialización no utilizada" fue el de mayor ocurrencia (44,6\%) y también el de mayor reducción (69,0\%). Conclusión: Las acciones educativas realizadas fueron efectivas para mejorar la calidad del proceso de codificación.

Descriptores: Grupos Diagnósticos Relacionados. Codificación Clínica. Sistema Único de Salud.

\section{INTRODUÇÃO}

O DRG - Grupo de Diagnósticos Relacionados (Diagnosis Related Groups) - é uma metodologia que mensura e categoriza a complexidade e a gravidade clínica de pacientes internados em 
hospitais. A combinação de dados como idade, diagnósticos principal e secundários, sistema fisiológico afetado e procedimentos realizados durante as internações são os parâmetros que determinam a complexidade e a gravidade clínica de pacientes. (COUTO et al., 2018). O DRG relaciona também essa classificação com os recursos consumidos durante o período de internação, criando categorias de pacientes que são semelhantes em suas características clínicas e no seu consumo de recursos. São considerados Isso possibilita a comparação e previsão de desfechos assistenciais e de consumo de recursos (NORONHA et al., I99I; MATHAUER; WITTENBECHER, 20I3).

A Fundação Hospitalar do Estado de Minas Gerais (Fhemig) formada pela fusão de três fundações estaduais de assistência à saúde (FEAL - leprocomial, FEAP psiquiátrica e Feamur- médica de urgência) em 3 de outubro de 1977, foi criada pela Lei Estadual 7.088. A Fhemig é uma das maiores gestoras de hospitais públicos do país, conta com mais de 12 mil profissionais e possui 2I hospitais que estão distribuídos em Belo Horizonte, região metropolitana e no interior do Estado. Tem como competência prestar serviços de saúde e assistência hospitalar de importância estratégica estadual e regional, em níveis secundário e terciário de complexidade. Todas as unidades assistenciais integradas ao Sistema Único de Saúde (SUS), participam da formulação, do acompanhamento e da avaliação da política de gestão hospitalar, em consonância com as diretrizes definidas pela Secretaria de Estado de Saúde de Minas Gerais (SES-MG).

Sistema DRG Brasil foi implantado na Fhemig, em 2019 o para análise do desempenho assistencial em dez dessas hospitais. O processo de codificação, implantado nesses hospitais em 2020, assegurou a organização de uma base de dados fidedigna que representa os serviços assistencias ofertados. Torna-se relevante mensurar o impacto das estratégias adotadas para avaliar a qualidade da codificação realizada na Fhemig. Por isso, no segundo semestre de 2019 foi iniciado o processo de auditoria das altas codificadas pelos $\mathrm{IO}$ hospitais. Este estudo pretende avaliar o impacto des ações educativas na qualidade do processo de codificação.

\section{REFERENCIAL TEÓRICO}

A classificação do DRG foi desenvolvida nos Estados Unidos da América na Universidade de Yale, no final da década de 60. Com o surgimento dos seguros Medicare (Programa norteamericano para assistência a indivíduos com doença debilitante ou maiores de 65 anos) e Medicaid (Programa social norte-americano para indivíduos de baixa renda) o governo norte-americano adotou a metodologia DRG para controlar o fornecimento e pagamento dos serviços hospitalares prestados (PAOLILLO et al., 2008).

O objetivo primário da metodologia dos DRG foi a definição de categorias de pacientes admitidos em hospitais que fossem homogêneas no consumo de recursos assistenciais para a solução de seus problemas de saúde. $\mathrm{Na}$ década de 80 , o DRG sofreu grande evolução metodológica tornando-se o sistema utilizado pelo governo americano em todo o seu território para avaliação do consumo de recursos para o tratamento hospitalar de pacientes agudos. A partir da década de 90, o uso do DRG foi disseminado em várias partes do mundo (Alemanha, Austrália, Espanha, França, Inglaterra, Itália e Portugal). Nesta mesma época foi incorporada à metodologia a avaliação de desfechos (como óbito, e complicações assistenciais) tornando o DRG uma ferrameta útil também para a eficiência da produção assistencial do hospitalar de pacientes com internações agudas (PAOLILLO et al., 2008).

A codificação clínica é o processo no qual profissionais de nível superior (analistas), treinados em codificação de saúde, através da análise de prontuários de pacientes, classificam as informações em saúde a partir da Classificação Internacional de Doenças (CID-I0). A atividade exige conhecimentos assistenciais e específicos relacionados à metodologia DRG.

A OMS, através da Aliança Mundial para a Segurança do Paciente, destaca a necessidade de desenvolver diferentes tipos de investigação para melhorar a segurança do paciente e prevenir os possíveis danos, em um ciclo de investigação que compreende: determinar a magnitude do dano, o número e tipos de eventos que prejudicam os pacientes, entender as causas fundamentais dos danos ocasionados aos pacientes, identificar soluções para alcançar uma atenção à saúde mais segura e avaliar o impacto das 
soluções em situações da vida real (NISHIO; FRANCO, 20I I). Paladini (2007) afirma que a qualidade é entendida pelo atendimento aos padrões técnicos estabelecidos e adotados em relação aos produtos ou serviços. Na perspectiva da adequação ao uso, refere-se à capacidade de satisfazer desejos do cliente e ao atendimento às suas necessidades. Neste sentido, o produto ou serviço é considerado de qualidade quando atende perfeitamente, de forma confiável e segura e no tempo certo, as necessidades dos clientes (PALADINI, 2007). Donabedian propõe uma sistemática para análise dos requisitos para a obtenção da qualidade assistencial, que se divide basicamente em três componentes: estrutura: implica na qualidade dos estabelecimentos e dos recursos materiais (como instalações, equipamentos e recursos financeiros) e os recursos humanos (número e qualificação do pessoal) bem como a estrutura gerencial (organização da equipe médica, métodos de controle e sistema de remuneração); processo: inclui as atividades ao buscar e levar a cabo a assistência e as opções ao fazer o diagnóstico e recomendar e executar um tratamento pelos profissionais da saúde; resultado: implica nos efeitos da assistência no estado da saúde do paciente e na sua aprovação aos critérios estabelecidos. Donabedian estabeleceu ainda os chamados sete pilares de sustentação da qualidade em saúde, quais sejam: eficácia, efetividade, eficiência, otimização dos recursos, aceitabilidade, legitimidade e equidade (DONABEDIAN, 2005). Este enfoque triplo da evolução da qualidade só é possível porque a adequada estrutura e processos aumentam a possibilidade de um resultado confiável. Donabedian define avaliação como sendo um processo que tenta determinar de forma sistemática e objetiva os impactos das atividades realizadas na melhora das condições de saúde do indivíduo. A auditoria como método de avaliação pode ser entendida como um conjunto de técnicas para verificação das estruturas, processos e resultados e a aplicação de recursos financeiros mediante a comparação entre uma situação encontrada e determinados critérios técnicos, operacionais ou legais, devendo-se avaliá-la objetivamente para determinar a extensão na qual os critérios da auditoria serão atendidos, sendo envolvidos neste processo o cliente da auditoria, o escopo de auditoria e o plano de auditoria. A auditoria independente é considerada a forma mais transparente de garantir qualidade para o paciente.

A auditoria é um processo contínuo e periódico que consiste em monitorar possíveis indicativos de falhas no processo de codificação, tendo como finalidade avaliar a qualidade da codificação, através dos seguintes critérios de acordo com Couto e Pedrosa (202I):

i. PÓS MDC: Pós-MDC é um termo estabelecido pelos desenvolvedores do DRG Brasil para melhor identificar os DRGs posicionados após o $25^{\circ} \mathrm{MDC}$, cuja característica é não haver correlação entre o CID principal e o procedimento cirúrgico realizado.

ii. Paciente sem DRG calculado: ocorre quando o sistema emite um alerta de que determinado CID não pode ser usado como CID Principal. Caso a mensagem de alerta seja ignorada, o registro é gravado mas o DRG não é calculado.

iii. CID Principal diferente do CID de CTI na mesma data: ocorre quando o CID Principal está diferente do CID do CTI dentro de 24 horas da admissão.

iv. CID secundário na condição adquirida: ocorre quando o CID Secundário está repetido na aba de Condição Adquirida.

v. Procedimento com especialização não utilizada: ocorre quando determinado procedimento possui topografias/especificações e o codificador não a especificou.

vi. CID com especialização não utilizada: ocorre quando o CID (principal ou secundário) possui topografias e o codificador não as utiliza para especificação, o que pode mudar o grupo de DRG.

vii. DRG 999/não definido: as informações lançadas durante o processo de codificação não se se relacionam com nenhuma Categoria Diagnóstica Maior (MDC).

\section{OBJETIVO}

Descrever o impacto de ações educativas na redução de inconformidades geradas pelo sistema Diagnosis Related Groups Brasil em I0 hospitais públicos de Minas Gerais, no período de junho de 2020 a junho 202I.

A Efetividade de Ações Educativas na Redução de Inconformidades Geradas pelo Sistema DR G-Brasil em IO Hospitais Públicos de Minas Gerais: Estudo de Caso 


\section{METODOLOGIA}

Trata-se de um estudo de caso sobre a efetividade das estratégias adotadas para reduzir a ocorrência de inconformidades de I0 hospitais públicos da FHEMIG, no período de janeiro a junho de 202I. O estudo de caso é útil para explicar, descrever ou explorar eventos ou fenômenos nos contextos cotidianos em que ocorrem. (ZHUANG et al., 2020).

$\mathrm{Na}$ FHEMIG, os analistas da CCDRG-FHEMIG realizam a auditoria das altas codificadas nos I0 hospitais a partir da emissão, via sistema DRG Brasil, de relatórios com as inconformidades. São emitidos, mensalmente, I0 relatórios (de cada hospital) onde estão descritos informações quantitativas e qualitativas dessas inconformadades apresentadas nas codificações realizadas. . Os dados desses relatórios (tipo e descrição das inconformidades e sugestão para correção das mesmas) são compilados em uma planilha de Excel ${ }^{\circledR}$ padrão pelos analistas da CCDRG-FHEMIG.

Para qualificar o processo de codificação, foram realizadas as seguintes ações educativas: treinamento sobre as inconformidades e reuniões mensais com apresentação e discussão com os dados das planilhas confeccionadas. É importante ressaltar que durante essas reuniões realizadas alguns analistas dos hospitais foram capacitados para o uso das de softwares (SIGTAP - Sistema de Gerenciamento da Tabela de Procedimentos, Medicamentos e OPM do SUS, PesqCID- programa de pesquisa por termos) e recursos do sistema DRG Brasil. Um treinamento intitulado "Verificação das inconformidades no processo de codificação" foi ministrado pela Central de Codificação DRG da FHEMIG (CCDRGFHEMIG) para os analistas dos I0 hospitais, no dia 05 de fevereiro de 202I, que abordou o conceito de cada tipo de inconformidade e as estratégias para corrigi-las. Durante o período do estudo, além do treinamento, foram realizadas reuniões mensais entre a CCDRG-FHEMIG e os analistas de cada um dos IO hospitais para apresentação e discussão dos dados das planilhas e esclarecimento de dúvidas sobre o processo de codificação. Previamente às reuniões, foram encaminhados, via Sistema Eletrônico de Informações (SEI) os relatórios de inconformidades e as planilhas com parecer e tratativas. Desta forma ocorreu a validação do processo auditoria.

Essa pesquisa foi realizada com dados dos relatórios e planilhas referentes às altas codificadas no DRG Brasil $\mathbb{R}$, no período de janeiro a junho de 202I. Foram descritas o quantitativo de altas codificadas e de inconformidades de IO (dez) hospitais públicos da Fundação Hospitalar de Minas Gerais (FHEMIG) que utilizam a metodologia DRG Brasil como ferramenta para mensuração de sua complexidade assistencial. Esses hospitais representam ambientes de cuidado diversos que atendem as necessidades de pacientes com diferentes perfis clínicos.

Para facilitar a descrição, compreensão e análise os dados foram quantificados e apresentados graficamente. A efetividade das ações educativas realizadas foram mensuradas a partir da comparação do número de inconformidades geradas pelo software antes e após a ação educativa.

Para o desenvolvimento desta pesquisa foram utilizados somente dados gerenciais, obtidos a partir de relatórios do DRG Brasil. Não foram utilizados dados diretamente de participantes ou informações que possam identificar os hospitais da FHEMIG que foram incluídos nesse estudo.

\section{RESULTADOS}

Um total de 23.326 altas foram codificadas no período de janeiro a julho de 202I. Observa-se um declínio inicial (no mês janeiro) e depois um aumento contínuo do número de altas codificadas, a partir de fevereiro. Por outro lado, nota-se o comportamento inversamente proporcional do número de inconsistências apontadas pelo sistema DRG-Brasil que reduzem durante o período de estudo, principalmente, após o mês de fevereiro. (Gráfico I).

Gráfico I - Relação inversamente proporcional entre o número de altas codificadas e de inconsistências 
apontadas pelo sistema DRG dos I0 hospitais, de janeiro a julho de 202I, Minas Gerais.

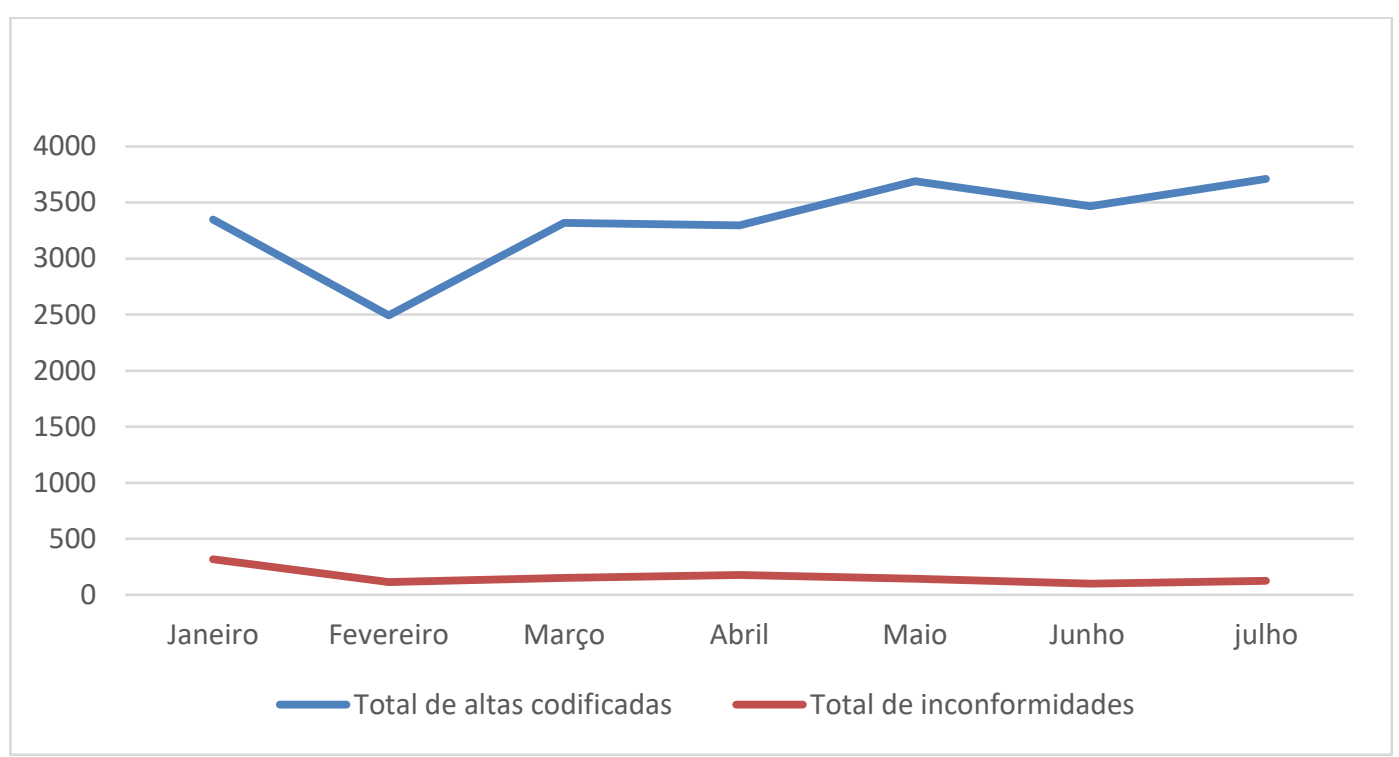

Fonte: Dados do estudo.

A partir das altas codificadas, foram identificadas I.122 inconformidades geradas pela codificação clínica no software DRG Brasil, no período do estudo, nas IO unidades hospitalares. Conforme representado no Gráfico 2, o número de inconsistências apresentou uma redução de 64,4\% entre os meses de janeiro $(\mathrm{n}=3 \mathrm{I} 7)$ e fevereiro $(\mathrm{n}=\mathrm{II} 3)$, quando foi realizado o treinamento sobre as inconformidades. Entre os meses de fevereiro a abril o número de inconformidades apresentou um aumento de $55,8 \%$ (n $=\mathrm{II} 3 / \mathrm{I} 76)$.

\section{Gráfico 2 - Inconformidades dos 10 hospitais no período de janeiro a julho de 202I, Minas Gerais.}

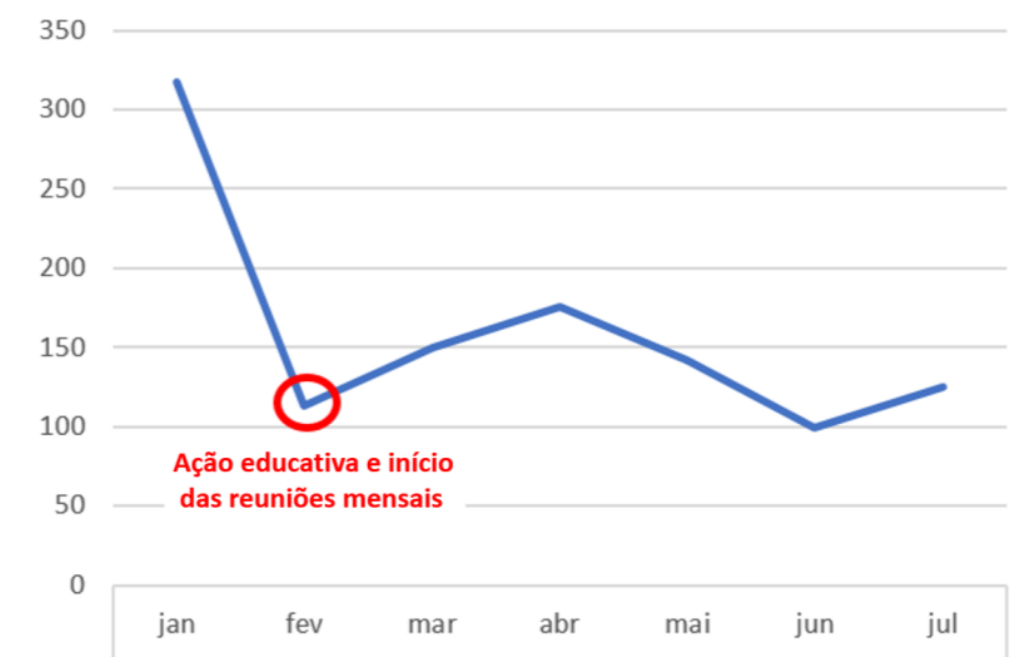

Fonte: Dados do estudo.

Dentre os tipos de inconformidades, o quantitativo e o percentual das mais frequentes foram, respectivamente, "CID sem especificação" ( $\mathrm{n}=53 \mathrm{I} / 47,3 \%)$, "Paciente sem DRG" ( $\mathrm{n}=$ I77/ I5,7\%) e "Pós-MDC" (n = I45/I2,9\%) e a menos frequente foi "DRG 999” ( $\mathrm{n}=35 / 3, \mathrm{I} \%)$ (Gráfico 3).

\section{Gráfico 3 - Quantitativo das inconsistências estratificadas}




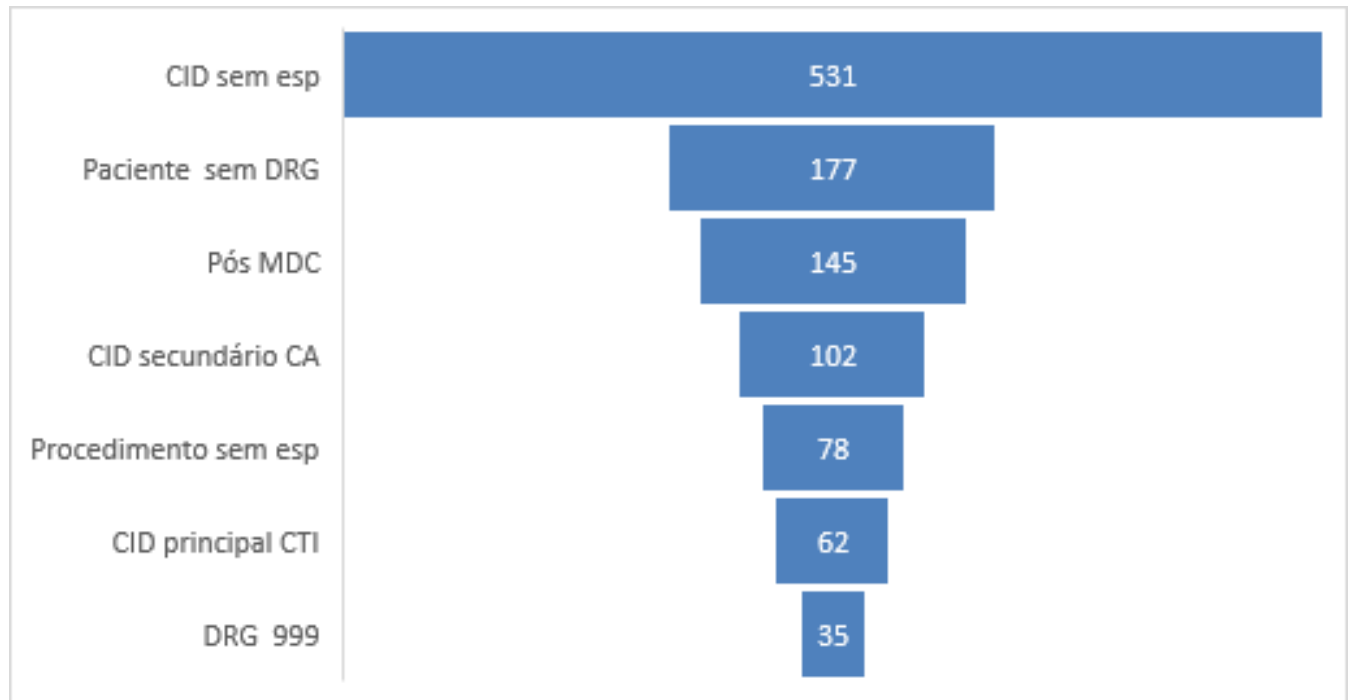

Fonte: Dados do estudo. Legenda: CID sem esp: CID sem especialização; Paciente sem DRG; Pós MDC; Cid secundário CA: CID secundário em condição adquirida; Procedimento sem esp: Procedimento sem especialização; CID principal CTI: CID principal diferente do CID CTI; drg 999.

Em geral, verifica-se um declínio importante do quantitativo das informidadedes nos 10 hospitais. Os tipos de inconformidades que apresentaram maior redução quantitativa entre os meses de janeiro e julho de 202I, foram respectivamente: $81,5 \%(n=38 / 7)$ "CID secundário repetido na condição adquirida", 69,0\% ( $\mathrm{n}=42 / \mathrm{I} 3)$ "Paciente sem DRG" e 65,0\% (n=I83/64) "CID com especialização não utilizada” (Gráfico 4).

\section{Gráfico 4 - Redução quantitativa das inconsistências estratificadas}

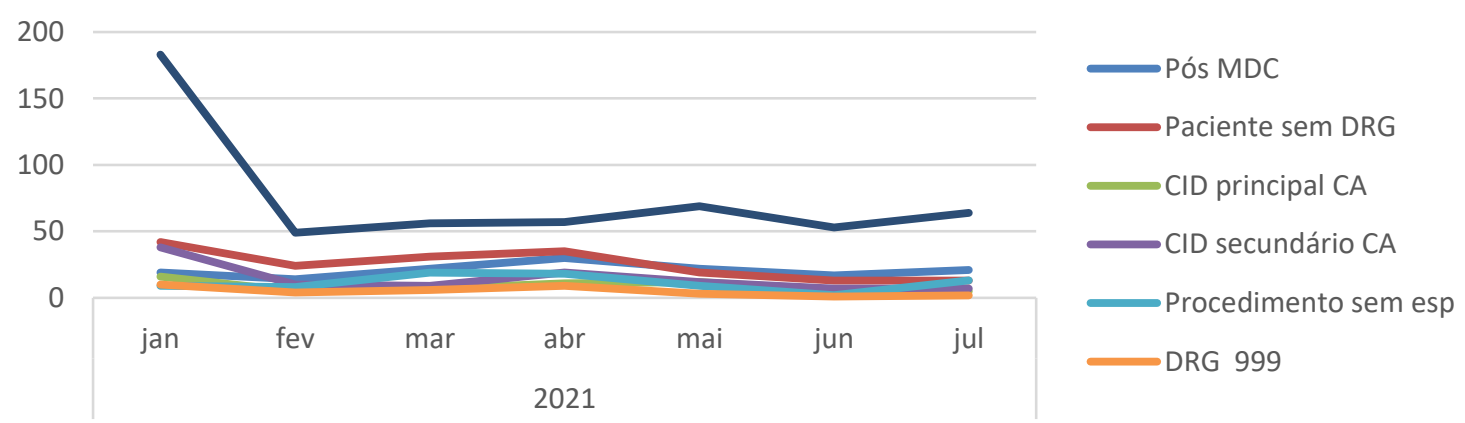

Fonte: Dados do estudo. Legenda: Pós MDC; Paciente sem DRG; CID principal CA: CID principal na condição adquirida; Cid secundário CA: CID secundário em condição adquirida Procedimento sem esp: Procedimento sem especialização; DRG 999, CID sem esp: CID sem especialização.

\section{DISCUSSÃO}

Do total de 23.326 altas codificadas nas I0 unidades hospitalares, no período de janeiro a julho de 202I, foram geradas, pelo sistema DRG-Brasil, I.I22 inconformidades. Após as ações educativas, o quantitativo dessas inconformidades apresentou redução durante o período do estudo, principalmente entre os meses de janeiro e fevereiro $(64,4 \%)$ quando os analistas dos 10 hospitais foram treinados sobre as incoformidades da codificação. Dentre os tipos de inconformidades, a mais frequente foi "CID com especialização não utilizada" (44,6\%) e a menos frequente foi "DRG 999" (3,1\%).

$\mathrm{Na}$ FHEMIG, o processo de codificação das altas iniciou, tanto na CCDRG FHEMIG quanto 
nos hospitais, no segundo semestre 2019. O crescente número de altas codificadas durante o período do estudo representa parte da curva de aprendizado de codificação e relaciona-se com o aumento progressivo do números de analistas. A queda do número de altas codificadas durante o mês de janeiro provavelmente se relaciona com o período de férias escolares quando comumente o número de internações diminui. Além disso, os analistas da CCDRG FHEMIG, que antes codificavam, direcionaram seus esforços para estruturação e execução da auditoria.

De maneira geral, o número de inconsistência diminuiu, mas é observada uma redução considerável de $64,4 \%$ logo após o treinamento realizado em fevereiro. Isso indica que esta ação educativa foi efetiva e capaz de qualificar as codificações realizadas nos IO hospitais. O aumento considerável do número de incondormidades ocorrido no mês abril (55,8\%), acompanha o maior número de altas codificadas no mês de março. Vale lembrar que o processo de auditoria das altas ocorre no mês posterior às suas codificações. Além do mais, a capacitação continuada e cobertura de férias dos analistas que codificam as altas também podem justificar oscilações do número dessas inconformidades.

A inconformidade mais frequente foi CID com especialização não utilizada (47,3\%) que está diretamente relacionada com a não utilização de CIDs específicos de acordo com a topografia o que pode mudar o grupo de DRG. Em segundo lugar está a inconformidade Paciente sem DRG calculado. Essa inconformidade relaciona-se ao não lançamento de dados obrigatórios em algum momento do processo de codificação impossibilitando o cálculo do DRG. E em terceiro lugar temos o Pós MDC (I2,9\%) que durante o processo de codificação não houve a utilização de ferramentas de pesquisa para se relacionar o CID ao procedimento descrito.

A efetividade das ações está diretamente relacionada às estratégias de capacitação utilizadas pela equipe. Reuniões individuais com os analistas de informação em saúde das unidades além da capacitação inicial foram cruciais para os resultados. Durante as reuniões há a possibilidade de entender melhor as dúvidas específicas individuais e realizar capacitações contínuas.

\section{CONSIDERAÇÕES FINAIS}

Os dados dessa pesquisa comprovam que as ações educativas (treinamento, análises mensais dos relatórios de inconformidades de codificação e os encontros para análise crítica e definição das ações de correção) foram realmente efetivas para melhoria da qualidade da codificação, reduzindo a taxa de inconformidades em $64,4 \%$.

Verificou-se na análise dos dados que o envolvimento da equipe de analistas de informação em saúde na qualificação dos dados codificados é condição essencial para o aumento da especificidade do registro de DRG, refletindo em maior precisão das análises do desempenho e qualidade assistencial.

O estudo possibilitou compreender que para aumentar a qualidade dos dados é necessário um trabalho contínuo de melhoria tanto da coleta das informações em prontuário, quanto da qualidade dos registros assistenciais, e que o uso das ferramentas, a adoção de técnicas e instrumentos e a capacitação de forma permanente são necessários.

Novos estudos precisam ser realizados nessa área para ampliar a definição de estratégias para mitigar as inconformidades nos registros de DRG. Não se pretendeu ao final deste trabalho esgotar todo o tema acerca das inconformidades dos registros de DRG e os mecanismos para qualificação dessa informação, todavia, o trabalho desenvolvido pela AT-DRG/Fhemig mostrou subsídios relevantes para qualificar as informações em saúde geradas pelo DRG.

\section{REFERÊNCIAS}

BUSATO, André; GEORG Von Below. "The implementation of DRG-based hospital reimbursement in Switzerland: A population-based perspective." Health research policy and systems, v. 8 p. 3I, 2010. Disponível em: https://doi.org/I0.II86/I478-4505-8-3I Acesso em: 3I ago 202I. 
COUTO, Renato Camargos; PEDROSA, Tania Moreira Grillo; ROBERTO, Breno Augusto Duarte; DAIBERT, Paula Balbino. DRG Brasil: transformando o sistema de saúde brasileiro e a vida das pessoas. Belo Horizonte: MG, 2018. Publicação digital (ePub). ISBN 978-85-47I-0I82-4.

COUTO, Renato Camargos; PEDROSA, Tania Moreira Grillo. Manual Operacional DRG Brasil® Módulos: Agrupador / Categorizador de Paciente. Belo Horizonte: MG, 202 I. Documento interno não publicado.

DONABEDIAN, A. The quality of medicine care: how can it be assessed. JAMA. I988; 260:1743-8.

DONABEDIAN, A. Evaluating The quality of medical care. I966. Milbank Q. 2005; 83(4):69I-729.

DRG - BRASIL. Introdução e Conceitos. Diretoria DRG Brasil. Versão: 02. Novembro 2020.

GAGNOM, D; NADEAU, S; TAM, V. Clinical and administrative outcomes during publiclyfunded inpatient stroke rehabilitation based on a case-mix group classification model. Journal of Rehabilitation Medicine. 2005; 37(I): p. 45-52.

NISHIO, EA; FRANCO, MTG. Modelo de Gestão em Enfermagem: qualidade assistencial e segurança do paciente. Rio de Janeiro: Elsevier; 201 I.

NORONHA, MF; VERAS, CT.LEITE IC; MARTINS, MS; BRAGA, Neto F; SILVER, L. O desenvolvimento dos" Diagnosis Related Groups"-DRGs. Metodologia de classificação de pacientes hospitalares. Revista de Saúde Pública. I99I; 25(3): p. 198-208.

PALADINI, EP. Gestão da qualidade: teoria e prática. 2.ed. São Paulo: Atlas; 2007.

PALMER, GR. International comparisons of hospital usage: a study of nine countries based on DRGs: School of Health Services Management, University of New South Wales; 1989.

PAOLILLO, E. CABRERA; D.MARTINS, L. SCASSO, A; CONSTATATIN, M; FERREIRA, M. et al. Grupos Relacionados por el Diagnóstico (GRD). Experiencia con IR-GRD en el Sanatorio Americano, sistema FEMI. Revista Médica del Uruguay. 2008; 24(4): p. 257-265.

WILEY, MM. Hospital financing reform and case-mix measurement: an international review. Health Care Financing Review. I99I; I3(4): p. II9-I33.

ZHUANG, Y. CHEN, YW. SHAE, ZY.SHYU, CR. Generalizable Layered Blockchain Architecture for Health Care Applications: Development, Case Studies, and Evaluation. J Med Internet Res. 2020 Jul 27;22(7):eI9029. doi: I0.2196/I9029. PMID: 32716300; PMCID: PMC74I80IO. 